\title{
The Extreme Edge at the Bottom of the Internet of Things: A Review
}

\author{
Jorge Portilla, Senior Member, IEEE, Gabriel Mujica, Member, IEEE, \\ Jin-Shyan Lee , Senior Member, IEEE, and \\ Teresa Riesgo, Member, IEEE
}

\begin{abstract}
The Internet of Things is being established nowadays, and the deployment of devices to be interconnected with each other and to the Internet is becoming a more common issue. The Internet of Things is structured in several levels and, in this regard, depending on the layer in which these devices are located, the complexity range and specific constraints arise. In the lowest level of this structure, the so-called edge is found, where the data are gathered from the environment by tiny electronic devices which are very limited in energy, computing, and memory resources. These devices, in turn, present different levels of complexity, which lead to a distinction between the edge layer and the sensor or extreme edge layer. In the extreme edge is where the most essential and limited tasks are carried out, these are sensing and sending data. Meanwhile on the edge, in addition to sensing, some processing can be performed in order to offload upper layers and to save precious energy by not communicating useless data. In this review paper, the structure of the Internet of Things is presented and the extreme edge is detailed, presenting the implementation options and requirements, with the purpose of having a better understanding of the needs when a specific application has to be developed and a set of devices has to be deployed. A special emphasis receives the bottom layer, focusing on devices oriented to last unattended for tens of years.
\end{abstract}

Index Terms-Extreme edge, edge computing, Internet of Things, wireless sensor networks, IoT devices, sensor layer.

\section{INTRODUCTION}

$\mathbf{T}$ HE Internet of Things (IoT) is one of the revolutionary paradigms of the beginning of the $21^{\text {st }}$ century. The term Internet of Things was coined by Ashton [1] in 1999 and recently has become one of the technologies to which everybody is looking at. Ashton's concept was related to how the information is generated and supplied to computers and the big opportunity that arises in giving control to the machines to collect and share with each other these data, with one main target: releasing the human being in doing tedious tasks like counting, tracking or checking, for the sake of reducing cost and waste.

The Internet of Things revolution has been linked to other revolutionary technologies of the last 30 years. The Mark

Manuscript received November 30, 2018; revised December 30, 2018; accepted December 31, 2018. Date of publication January 10, 2019; date of current version April 5, 2019. This work was supported by the Ministry of Education, Culture and Sports of the Government of Spain, within the program Jose Castillejo for post-doc research stays, under Grant CAS17/00259. The associate editor coordinating the review of this paper and approving it for publication was Prof. Huang Chen Lee. (Corresponding author: Jorge Portilla.)

J. Portilla, G. Mujica, and T. Riesgo are with the Centro de Electrónica Industrial, Universidad Politécnica de Madrid, 28006 Madrid, Spain (e-mail: jorge.portilla@upm.es; gabriel.mujica@upm.es; teresa.riesgo@upm.es).

J.-S. Lee is with the Department of Electrical Engineering, National Taipei University of Technology, Taipei 10608, Taiwan (e-mail: jslee@ mail.ntut.edu.tw).

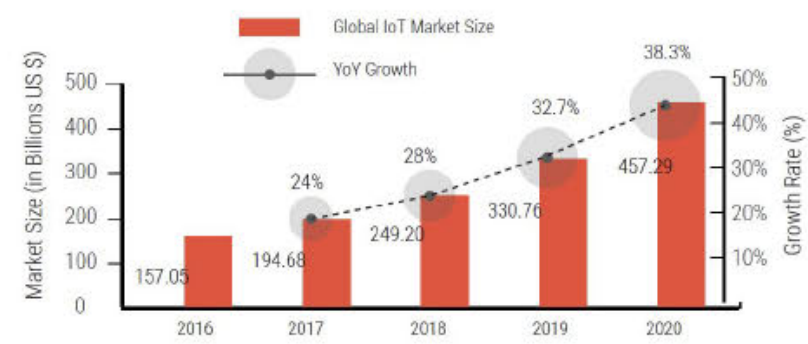

Fig. 1. IoT market growth prevision [6].

Weiser's concept of Ubiquitous Computing [2] established the basis for what was about to come. In this context, Wireless Sensor Networks (WSNs) have been the realization of the Ubiquitous Computing dream during the last three decades, where a myriad of low-end computers populate the environment transparently, carrying out processing tasks for a plethora of applications and communicating data among each other. These Wireless Sensor Networks can be seen in many cases as the physical connection of the IoT with the world. The IoT is of course made of things and the WSNs are part of this set of things.

The importance that the IoT has acquired can be sensed by available market forecasts. According to Statista [3], approximately 30 billion of IoT devices will be connected by 2020 . This number varies depending on the source. Ericsson [4] foresees around 25 billion devices in 2020 and Gartner [5] 20 billion by the same year.

In this context, according to [6] the global IoT market is set to grow from US 157 billion US dollars in 2016, to US 457 billion US dollars by 2020 (see Fig. 1). In this report it is also mentioned that SoftBank invested 32 billion US dollars to acquire ARM and expects to produce a trillion IoT chips over the next 20 years. In the same line smartwatches and fitness wearables dominate investment and accounted for over $70 \%$ of total wearables sales worldwide in 2016. Companies such as Bosh have created a procedure model for developing business models for IoT offerings [7] due to the relevance that the topic is nowadays gaining.

At this point, it becomes necessary to establish what a thing is. In the big picture of the Internet of Things, several elements (things) can be noticed, as phones connected to each other and to the Internet, cars, house appliances notifying their status to the owner through the Internet, or street lights that turn on and off remotely or by the measurement of a sensor, just to give a few examples. These elements are very different from each other, but all of them are things to the IoT. From a general 
TABLE I

CLASSIFICATION AND NUMBER OF THINGS IN BILLIONS AND THE COMPOUND ANNUAL GROWTH RATE FROM [4]

\begin{tabular}{|c|c|c|c|}
\hline & 2016 & 2022 & CAGR \\
\hline Wide-Area IoT & 0.4 & 2.1 & $30 \%$ \\
\hline Short-Range IoT & 5.2 & 16 & $20 \%$ \\
\hline PC/laptop/tablet & 1.6 & 1.7 & $0 \%$ \\
\hline Mobile Phones & 7.3 & 8.6 & $3 \%$ \\
\hline Fixed Phones & 1.4 & 1.3 & $0 \%$ \\
\hline
\end{tabular}

perspective, these elements do not possess any special feature that allows them to be part of the Internet, any electronic component or communication module, in principle.

Hence, a thing in IoT is a networked embedded computer with some sensing capabilities, able to communicate, mainly wirelessly and mainly through the Internet (not always). These embedded computers are usually but not necessarily attached to daily life objects as the ones mentioned before. The big contribution of the Internet of Things to the human kind is the availability of infrastructures, both hardware and software, for massive distributed data collection and processing, which may help in uncountable problems solving from world energy consumption reduction or city traffic optimization to catastrophe effects minimization or attack prevention.

The different nature of the daily life objects set different requirements for the embedded computers within. In this regard, the classification made in [4], which can be seen in Table I, established five types of things attending to the processing and communication resources of these things. As it can be seen in Table I there will be an explosion of the Widearea IoT and the Short-range IoT in the near future, considering the compound annual growth rate (CAGR) expected. These Wide-area and Short-range IoTs are another way to call the Wireless Sensor Networks. If the forecast made by Ericsson becomes true, and the authors of this paper are aligned with this idea, this means that billions of these so-called IoT devices will be deployed before 2025 .

As it has been mentioned before, the Internet of Things is a mixture of hardware and software components, ranging from the smallest 8-bit microcontroller-based IoT device to the most powerful supercomputer in the cloud. This heterogeneity and the way data are generated, stored and processed set different layers for the IoT, that will be analyzed along this paper, focusing on the lowest layers.

The main contribution of this review paper is to give clear idea of the needs for a realistic Internet of Things realization, considering that perpetuity and autonomy will be key aspects to consider in the devices located in the bottom layer of the IoT. From the cloud to the extreme edge, different technologies and frameworks are and will be demanded, but is in the connection with the world where the scene is not clear, in order to have billions of deployed devices supplying data in an unattended way. In this paper, the state-of-theart research works in this sense are presented and discussed, and the requirements for the sensor layer of the IoT are derived. In this regard, a classification of the IoT devices in the edge is presented.

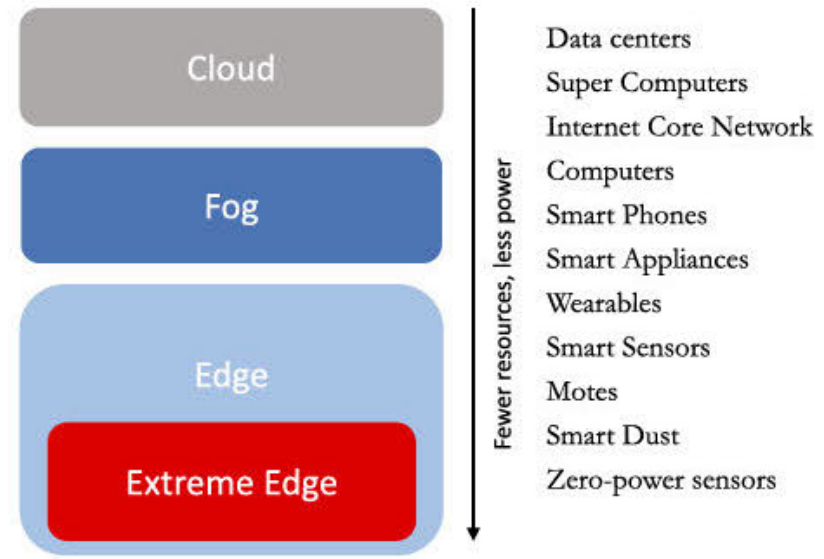

Fig. 2. Structure of the Internet of Things.

There are still many research challenges to make the paradigm of 20 billion deployed devices a reality. Issues related to energy harvesting technologies, ultra-low power devices and better and more efficient communication protocols are to be addressed.

In summary, the main contributions of this review are:

1) Present the most accepted structure of the IoT by the scientific community.

2) Analyze the lowest layers of the IoT, this is the Edge and the Extreme Edge layers.

3) Describe the requirements for realistic IoT deployments, concerning perpetuity and autonomy in the Extreme Edge.

The rest of the paper is organized as follows: in Section II an overview of the layers of the Internet of Things is given from different perspectives. In Section III, the edge layer is detailed and Section IV includes a review of the lowest layer of the IoT, the sensor layer. In Section V an analysis of the requirements and future trends for IoT devices is presented, whereas in Section VI conclusions and future perspectives are highlighted.

\section{The Structure of the Internet of Things}

The IoT relies on a set of well-known established technologies plus other novel ones, and is divided into different layers. This division presents different approaches depending on the point of view used to classify the IoT. In this section, the fundamentals and structure of the IoT are presented, to introduce the basics that will drive this review to more detailed issues regarding its lowest layers.

As it has been stated previously in this paper, the IoT is a combination of hardware and software to generate massive data gathered for different applications. These HW and SW components are wide-ranging, from resource-constrained sensors with short-range low-rate communication technologies to supercomputers in the cloud. This is what has been called the cloud-to-things continuum [8].

Reviewing the literature, a set of layers are usually defined when the IoT is described. Usually, four layers are distinguished (see Fig 2), but it must be said that there is no consensus on this issue. Even these layers are sometimes fused, resulting in a three-tier or two-tier model. 


\section{A. Cloud}

According to The National Institute of Standards and Technology (NIST) [9], cloud computing is a model for enabling ubiquitous, convenient, on-demand network access to a shared pool of configurable computing resources (e.g., networks, servers, storage, applications, and services) that can be rapidly provisioned and released with minimal management effort or service provider interaction.

This general definition shows the power that cloud computing makes available to the Internet of Things. The basic idea is to collect information, usually big amounts of data, in the point of interest (this so-called the edge, where information is generated) and upload it to be properly processed in the cloud, where ideally permanent and enough processing resources are available. This model may work perfectly with some approaches where the energy resource is not a limitation or the time required for any decision does not impose a very fast reaction.

However, the cloud computing paradigm applied directly to IoT presents a set of drawbacks regarding latency, bandwidth and storage [10] because of the huge amount of data that have to be uploaded and processed. Due to these limitations, more layers have been proposed during the last years, getting closer to the source of data.

\section{B. Fog}

Bonomi et al. [11] at Cisco coined the concept of Fog computing as a highly virtualized platform that provides compute, storage, and networking services between end devices and traditional Cloud Computing Data Centers, typically, but not exclusively located at the edge of networks. Another definition of Fog is that it is an architecture that distributes computation, communication, control and storage closer to the end users along the cloud-to-things continuum [8]. NIST defines fog computing as a layered model for enabling ubiquitous access to a shared continuum of scalable computing resources [12].

Perhaps, the use of the name fog attended to marketing purposes. The fog shares many similarities with another concept: the edge, which usually leads to confusion or makes them be put on the same level. Even some authors consider fog and edge as the same concept, as in [13].

The Fog has in its very heart the approximation of cloudlike resources as processing and storage closer to the data source. In the Internet of Things, this means bringing the cloud closer to the sensors and IoT devices. Moreover, the fog has the capability of sending selectively data to the cloud under request or based on certain rules.

\section{Edge}

According to [10] Edge computing refers to the enabling technologies allowing computation to be performed at the edge of the network, on downstream data on behalf of cloud services and upstream data on behalf of IoT services.

One of the most fundamental characteristics of the edge is that the processing, networking and storage are made right in the IoT devices or the sensor nodes, meaning that in edge computing, the communication with the cloud is not mandatory. Due to this, one key difference between fog and edge is that in fog computing the processing is carried out in more powerful devices than IoT nodes, as Internet gateways for example. Instead, the edge is decentralized by nature, allowing autonomous decisions [14].

The nature of the processing resources of the edge nodes allows a secondary division: edge nodes and extreme edge nodes, being the last ones those powered by batteries or energy harvesters and very limited in computing and storage capabilities to reduce costs and enlarge lifetime. In this type of devices, the ideal situation is to get perpetual systems avoiding battery replacement. In this context the term perpetual refers to a lifetime of tens of years, considering that ideal perpetuity is unfordable due to technology or degradation issues. In this regard, perpetuity will be referred to as very long-term lifetime for the sake of correctness along the rest of the paper.

\section{Extreme Edge (Also Known as Mist or the Things)}

The bottom layer of the IoT is the one in charge of taking measures from the world. Usually, this action is made by tiny devices composed of sensors, a low-end microcontroller, a radio module and a battery or/and an energy harvester, these are the things.

These devices, in many cases, can connect to each other creating a short area network (Personal Area Network, PAN, tens of meters) or wide area network (Long Range, tens of kilometers), frequently using unlicensed radio frequency bands as the ISM (Industrial, Scientific and Medical) ones. This has been the approach of the wireless sensor networks during the last 20 years, which now become part of a bigger reality that is the IoT.

Some authors have named this layer as the Mist [15], stating that Mist computing pushes processing even further to the network edge, involving the sensor and actuator devices. Preden et al. [15] affirm that Mist computing reduces latency and increases autonomy, and self-awareness of every device becomes crucial as the computation and actuation depend on the environment. This Mist approach is, in other words, taking networked embedded systems as wireless sensor networks as the lowest layer of the IoT. In Fig. 2 the layers of the IoT are shown along with the domain of the processing elements and devices in each layer.

At this point, the paper enters into detailing the edge of the IoT, distinguishing two layers inside: the edge and the extreme edge. The analysis and discussion presented in the next sections are the main contributions of this review paper.

\section{The EdGE}

With the progressive growth of heterogeneous and more powerful devices on the Edge, it is clear that the decentralized nature of this layer shall be taken as an advantage to provide runtime distributed processing and dynamic decision-making capabilities, so that a meaningful communication with the Fog and Cloud layers are performed (only if needed). Unlike traditional WSNs where sensor nodes get measurements from the environment under monitoring and then transmit such data to remote sinks, the Edge Computing is envisioning one step further in highlighting the collaboration among deployed devices, so as to produce significant information 
directly on-site. In this context, an efficient coordination of Edge and Extreme Edge technologies will certainly leverage the overall self-management of IoT devices. This is particularly important considering two main issues to be tackled, i.e., 1) data gathering from and management of such a huge amount of devices and 2) energy provision to assure their long-term operability and maintenance.

\section{A. Edge Computing}

The traditional node architectures, where 8-bit microcontrollers were the main core of the sensor platforms for performing simple acquisition tasks, are evolving towards more sophisticated microprocessors and System on Chip that provide enhanced trade-off solutions between processing capabilities and power consumption [16]. However, since the effective duty cycle and energy demand of these devices are also increasing in line with their computational load (and thus the network lifetime will anyway be penalized), they are rather planned to be deployable platforms with accessible external power sources in most of the real application cases. This approach goes aside the Extreme Edge, where different strategies are upon the table to achieve perpetuity with no outer power supply, as detailed in subsequent sections of this paper.

As mentioned before, it is sometimes diffused the border line between Edge and Fog computing in the state of the art, depending on the approach followed to identify the different components of the IoT technologies at different levels. This is the case of Multi-access Edge Computing paradigm [17] which has been thought to close the gap between the Cloud service functionalities and the network edge devices, offering a lower end-to-end latency with a better traffic performance. Similarly, the concept of Cloudlets [18] tries to bring the Cloud computing and storage closer to mobile devices so that the interaction delay between the service consumer and service provider becomes shorter. Instead, in this work the Edge rather contemplates possible "Cloud-less" contexts where the processing autonomy of the end devices is empowered by the collaboration among participant IoT Edge nodes, and their relationship within the network.

\section{B. Heterogeneity on the Edge}

Following the aforementioned ideas, mobile devices (such as smartphones or next-generation wearable devices) are considered as one of the groups that compose the Edge Computing (particularly within the participatory sensing picture [19], where users become data providers as well), while sensor platforms can be seen as permanent city/industrial metadata producers and they additionally provide the underlying technologies for fully supporting the on-site decentralized IoT infrastructure on the Edge. In general, when referring to more powerful Edge sensor platforms, it usually comes in mind the wide-spread Raspberry Pi board, which certainly fits within the IoT gateway side (much closer to the Fog). However, System-on-Chip based embedded platforms are clearing the horizon towards consolidating the present (and the near future) of IoT edge device architectures. In this regard, ARM-based MCUs with radio transceiver integrated on chip are currently being supported in most of the IoT embedded operating systems (in particular Contiki-NG, RIOT and Linux-based OS) offering important updates for efficient power management, task scheduling, and IP-based networking capabilities [20].

One step further in strengthening the baseline conception of the decentralized IoT Edge is related to tackling the crossconnectivity issues that undoubtedly arise when having heterogeneous technologies under its umbrella. Although several approaches are independently appearing in the literature, some authors propose hardware-software frameworks for both IoT embedded application development and seamless integration of sensor platforms (such as direct synergies between smart wearables and WSN nodes, proposed in [21]), as an intermediate solution seeking the next-generation of distributed middleware [22]. This is particularly important for the actual implementation of modular and reusable intelligent components that can support the generation of significant data on the Edge nodes.

\section{Addressing Distributed Applications on the Edge}

Combining the increasing computing capabilities on the Edge with the energy-efficient sensing potential of the Extreme Edge will indeed make the approach of distributed applications a more feasible strategy to exploit the collaboration among the participant IoT nodes. In general, the effective coordination of embedded distributed functionalities of these types of applications has been linked to the capabilities of the Fog layer and, therefore, it serves as the gathering integration point to further correlate the data produced by the traditional sensor nodes. With the evolution of the Edge Computing and more particularly with the pronounced improvement on the processing and power management techniques, the possibility of implementing distributed computing techniques on the Edge has become a reality. This means that intelligence on the edge can be actually applied without losing a resource-constrained awareness of the bottom layer.

The issue of distributed applications on the Edge not only regards the hardware requirements to make possible the implementation of algorithms, but also encompasses communication and networking strategies to facilitate the cross-connectivity among deployed devices. As such, the multi-hop communication is indeed a must, but dynamic mesh networking or at least clustering techniques upon the wireless low-rate communication channel shall be the baseline techniques to promote collaborative contexts on the edge.

On the other hand, there are remarkable advances on proposing new paradigms for exploiting distribution in IoT, as the approach proposed in [23] where a programming model for large-scale distributed sensor network applications is addressed by the application model that maps mobile fog processes into distributed computing instances, allowing datastream handling from the edge devices.

However, when analyzing the main trends for addressing distributed applications on the edge, two general streams can be found in recent state-of-the-art proposals: Distributed middleware and Agent-based Computing Paradigm (the latter can be even realized with the goodness of the former one). The approach of integrating middleware capabilities on the Edge in an embedded form allows enhancing dynamic self-discovery 
and context-aware capabilities to be shared among local nodes, which then provides an enhanced coherence model of the local computing with respect to the overall system. Moreover, there are contributions in the literature related to the creation of cooperative connected smart objects in the form of middleware-driven intelligent multi-agent systems [24], with the support of one or more communication models (for instance, oriented to distributed publish/subscribed mechanisms). Additionally, there are more advanced solutions towards distributed smart objects on the Edge with the combination of cognitive multi-agent systems for heterogeneous deployments [25], where middleware capabilities bridge the management and programming of such objects in large-scale distributions.

However, as shown in the following section, the feasibility of applying these techniques to the Extreme Edge side is limited due to the intrinsic nature of the involved technology, that is, the provision of sensing or triggering actions under zero-power conditions. Thus, in general, it is not an approximation for the very bottom part of IoT but rather a mechanism to be applied right on top of it, though taking advantage of its sensing and context related features.

\section{Industrial Applications on the Edge}

Edge computing has gained attention in the last decade and the industry is using it as a valuable approach for solving different problems. In this regard, researchers in [26] present a platform concept, which combines cloud computing and industrial control using edge devices realized for an automation cell. The target is lying behind the approach of industrial control of field devices, comprising self-contained systems in a dedicated network for exchanging control information between field devices and control hardware, so as to accomplish process tasks and moving towards a solution based on the continuum cloud to edge.

Moreover, the new Industry 4.0 revolution is putting more load in all the layers of the IoT, from the edge to the cloud. In this context, researchers present in [27] the multitier multi-access edge computing (mMEC) as one of the most important technologies within 5G. The internetworking model in the smart factory is analyzed, and then each edge node is mapped into the mMEC platform to identify its computation performance.

\section{The EXTREME EdGE}

Some authors have already classified the IoT devices like in [28]. However, very few attention is being paid to the extreme edge devices focusing more on what it has been the usual approach in the last two decades, that refers to a low-end low power 8-bit or 16-bit microcontroller, a radio transceiver using a low data rate short-range communication protocol as IEEE 802.15.4, and powered by standard coin cells or AA batteries. The leader in this type of HW approach has been and is the TelosB platform, developed in 2005 at U.C. Berkeley [29].

These types of nodes have been very popular for testing different concepts in the area of WSNs, because of their availability, price and standardization in the way of software support and a community behind them. Very good examples of this fact are the operating systems TinyOS [30] and Contiki [31] that have been the niche for plenty of research works relying on the well established library of components and testbeds available.

However, in this type of devices as TelosB, there is one critical aspect that has to be considered and needs full attention, this is the perpetuity of the sensor deployments to avoid energy source availability problems and battery replacement in a "billion devices" paradigm. One of the most important issues that has to be taken into account is the energy supply to the devices. In [32] a study of the TelosB is made, confirming that this HW platform consumption ranges from $500 \mu \mathrm{A}$ to $25 \mathrm{~mA}$ approximately, which roughly means that in the best case, with standard AA batteries, assuming a charge of $1 \mathrm{Ah}$, the nodes can last for 3 years with very low duty cycles, in the order of $1 \%$, which means being awake 3,6 s per hour. Even with bigger batteries, the situation is not the desirable one for a very long-term lifetime deployment. This means that in many cases, deployed nodes with this power consumption profile will need to be connected to the mains or will need another type of energy source, probably a harvester, able to supply power in the order of a hundred of milliwatts. On the other hand, for such a low profile performance, the size of this device is still big, in the order of $20 \mathrm{~cm}^{2}$.

A very good example of the limitations of this type of nodes is shown in [33], where a low power microcontroller TI MSP430F5438A along with a CC2420 for IEEE 802.15.4 standard are used. This architecture is very similar to the TelosB one. Authors of this work use eight solar cells for energy harvesting purposes with $89.2 \mathrm{~mW}$ of peak energy at $2 \mathrm{~V}$ each one. Authors fail in getting perpetuity with this setup, for an always-on approach.

Even though there are strong limitations for this type of devices, a proper combination with the edge could bring benefits in terms of power savings and performance improvement.

For the aforementioned reasons, the sensor nodes of the IoT must be scaled down in resources and thus in power consumption. Moreover, the nature of the power supply has to tend to harvest energy from the environment to make the IoT sensors autonomous to the maximum extent as possible. Therefore, TelosB and other wireless sensor nodes as [34] or the ones compared in [35] may be used as prototyping platforms or edge low-end devices, always keeping in mind the energy limitations of such devices. The approach should be closer to the pioneer one that was presented in 1999 coining the concept of Smart Dust and motes [36]. This approach was very good in terms of integration and autonomy, but presented an important drawback that was the necessity of the line of sight for communication between motes because the laser was the chosen technology for this purpose.

With all these considerations, in this section, a review of sensor nodes for the extreme edge is presented, focusing on those tiny nodes or motes oriented to very specific tasks with very limited processing and memory resources, and with limited energy budget ant their energy constraints and supplies. 


\section{A. Edge Nodes}

As it was mentioned, in the IoT structure different layers can be identified. In the lowest level, the edge is present. It is so-called edge because it is the closest level to the source of data, the physical magnitudes. Sensors and actuators are located here. However, there is a fuzzy limit between the fog and the edge and, in this regard, there are even more levels of complexity that drive to divide the edge into those devices intended for measuring and communicating and those ones that may carry out more processing intensive tasks [37].

What is a more common tendency is bringing more intelligence to the smart sensors, which is necessary for the global Internet of Things integration [38]. The sensor layer of the IoT is the one that will count on the highest amount of devices (billions in principle), and these devices will be deployed everywhere, most of the time unattended. Moreover, these smart sensors will communicate to each other mostly with radio transceivers, using protocols not always directly integrated with the Internet [39].

Due to the huge number of devices that will be deployed in the near future, replacing batteries cannot be an issue anymore. In this regard, the scientific community is working on improving both efficiencies of the HW for sensor nodes and battery capacity, together with better energy harvesting techniques and algorithms for decreasing power consumption.

The autonomy of the IoT deployments in terms of energy arises from two points: the first one is the need for avoiding battery replacement even in very low duty cycle applications. The second one is the existence of applications that need continuous sensing as the ones related to the assisted living context or critical systems. In [39], authors face this scenario by implementing policies for energy optimized sensor activation, getting good improvement results comparing with no optimization implementations, reducing battery spending three times in the best case and achieving 175 hours of lifetime, for an elderly fall detection system. However, even though these results are good, battery recharging or replacing is still mandatory. Their results are good as a testbed for future optimizations in the context of activities of daily living sensing.

In the context of wearable devices for health care, Basu et al. [38] present a solution for smart wearable devices. In this case, the authors take advantage of the inherent tasklevel parallelism of the bio-signals. In this work the target is the inclusion of a coarse-grained reconfigurable architecture made of several RISC (Reduced Instruction Set Computer) processors interconnected by a configurable matrix, synchronization schemes for SIMD (Single Instruction Multiple Data) execution and inexact computing model. Anyway, the target for this architecture is still far from the perpetuity, although very good results are obtained due to the complexity of the system.

Following the approach of Smart Dust, in [40] a sensing platform with energy harvesting is presented. The size of the mote is $1 \mathrm{~mm}^{3}$ and it is a very good example of what a mote should look like in terms of size and power consumption. They present a stacked architecture with two processors, sensors and a Li-ion battery that can be recharged by solar energy harvesting. It also includes an optical communication module for programming after assembly, synchronizing and reprogramming if necessary.

\section{B. Smart Dust}

The previous examples are very good candidates for sensor nodes in the edge close to the extreme, and they are highly resource-limited sensor nodes. Even though these type of devices will exist by billions in the IoT, there exist space for other sensor nodes with more power consumption but still with access to perpetuity. This is the case of the node presented in [41] in which a node for snail detection in an agricultural environment is presented, in order to avoid pests that may damage the plantations. Perpetuity is necessary for the purpose of the application, and radio communication is a requirement as well. In this case, power requirements are higher, but there is also the possibility of including bigger solar cells to cope with the energy demand. The hardware of this device is based on commercial radio and microcontroller modules. In this case, a solar panel supplying $100 \mathrm{~mA}$ and $5 \mathrm{~V}$ is used, taking advantage of the space size and that plenty of sunlight is available. The results show that one hour is enough to recharge the batteries (1000 mAh) so probably the hardware could be scaled down to decrease price.

The examples shown up to now exposes a direct relationship between size and energy availability. In the IoT paradigm, many of the deployed devices will have to cope with the restriction of non-intrusiveness, meaning that small size is mandatory, in the order of some $\mathrm{mm}^{3}$. This is the main challenge, coping with reduced size and maintaining performance with very low power consumption. According to [42], the power budget for a multiyear operational lifetime is in the order of $1-10 \mathrm{nW} / \mathrm{mm}^{3}$. The main reasons for these limitations are the harvester's energy generation capabilities and the battery capacity. Focusing on wearable IoT devices, energy harvesting from the human body with vibration or thermal sources is in the order of $2.2 \mu \mathrm{W} / \mathrm{cm}^{2}$ for thermoelectric generators and $7.4 \mu \mathrm{W} / \mathrm{cm}^{2}$ for piezoelectric generators, meaning that suitable sensor node design could fit in this type of IoT devices for always-on performance.

\section{Zero-Power Sensors}

Continuing with the topic thread of the need for measuring in every place possible, it is clear that sensor nodes must scale down even more to be able to work unattended in inaccessible locations. In this point, the zero-power sensing concept is proposed by Qian et al. [43], where all the energy needed to produce the data of interest is generated by the specific physical phenomenon that is being measured, with no other power supply present in the system. This is a breakthrough for achieving always-on things in IoT. The basic idea is replacing the electronics that are consuming power continuously, even in very low power modes, by micro-electromechanical relay technology. This technology uses mechanical contacts between terminals instead of transistors to set or not a signal, therefore the standby power consumption due to sub-threshold conduction is practically eliminated (the energy in a switching cycling is in the order of attojoules, reported in [44]). 
In this line, the work reported in [45] is a demonstration of an infrared wireless sensor node with near zero-power consumption, using MEMS (Microe Electro Mechanical Systems) photoswitches. Authors use the sensor to wake up a node when the signal of interest is detected. The node is powered with a coin cell. The sensing element takes $2.6 \mathrm{nW}$ to generate a wake-up signal to the WSN node. The WSN node is consuming $180 \mathrm{nW}$ while sleeping. In this situation, the node can last more than ten years, only waking-up when the sensor detects any event of interest, and therefore, depending on this, the battery duration may change considerably. Anyway, to the best of the authors' knowledge, this represents the first implementation of a sensor node with zero-power sensors, that are always one expecting for a specific event, that can wake up a mote that is powered by a commercial battery, without any harvester or other power supply, and can last for years without missing any event, everything working together.

An application using zero-power sensing is presented in [46], targeting underwater monitoring using sound sensing. Authors achieve always-on event-driven audio detection for pattern recognition detection, consuming $55 \mu \mathrm{W}$ of power. They use a mixed approach with the event-driven detector and a microbial fuel cell that makes use of microbes in the soil to generate energy. Therefore, a zero-power system is achieved with no batteries or other power sources for the sensing system.

The DARPA N-Zero program [47] is seeking for zero-power sensors and signal processing that allow systems to last just using the self-discharging power of a battery, which allows lifetimes in the order of tens of years. This system will select an event from a noisy environment and will wake up a higher order system based on detected events. The target is to achieve sensor networks deployments that can last for decade-long lifetimes.

A military focused work is presented in [48], where piezoelectric MEMS accelerometers tuned for a specific frequency are used to detect intruders in an area of interest. The system is composed of the sensor plus a CMOS (Complementary Metal Oxide Semiconductor) stage to generate a wake-up signal, achieving consumptions in the order of $5.4 \mathrm{nW}$, needing to supply energy to the CMOS comparator. This is called near or quasi zero-power sensor.

A very good approach is the one presented by Pinrod et al. [49], where a combination of zero-power piezoelectric movement sensors and quasi zero-power NEMS (Nano Electro Mechanical Systems) switches are used to create a trigger-bit device that can wake up a more powerful sensor node (as a TelosB, as they illustrate). Their approach is intended to measure acceleration, magnetic field and orientation by detecting patterns using lead zirconate titanate (PZT) piezoelectric sensors. They get $5 \mathrm{nW}$ of power consumption combining the sensors and the NEMS switches that generate the wake-up signal.

Even though the aforementioned works are very promising, there is still a place for CMOS implemented smart sensors, as the temperature sensor presented in [50]. In this work, a smart temperature sensor fully implemented in CMOS technology is presented. This approach is the one related to

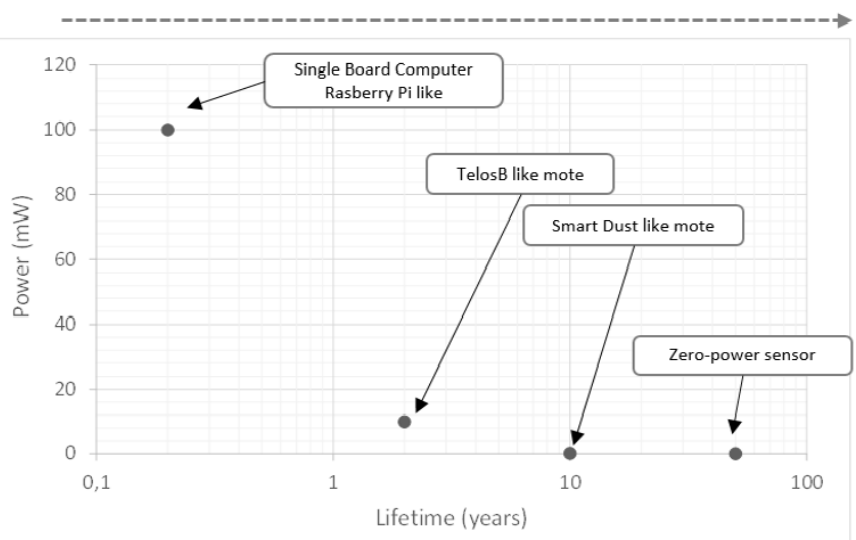

Fig. 3. Edge and extreme edge nodes power vs lifetime.

smart sensors, where the device includes the sensing element plus the necessary electronics to supply proper digital signal to a secondary circuit that will carry out signal processing and other tasks. In this work, the authors get around $80 \mathrm{pW}$ of power consumption in the worst case, which makes this sensor suitable for very long-term lifetime IoT applications. With the proper energy source the perpetuity is affordable using this sensor. In Fig. 3 the orders of magnitude of the different aforementioned types of edge and extreme edge nodes are displayed.

All the examples described in these previous subsections use different energy sources, depending on the specific application requirements and the particularities of the technology. In this context, the nodes in the type of the Single Board Computers and the TelosB like platforms may use batteries or connection to the mains. Eventually, dedicated harvesters can be included if the application duty cycle is short enough. Approaching to the extreme edge, the sources are mainly harvesters, from the environment or from the proper signal that is being taken. Independently of the energy source, that may drive to specific power supply circuits, the analysis has been made considering only the unique features of every set of devices.

\section{Communications in the Extreme Edge}

At this point, it is applicable to point out the benefits and drawbacks of optical communications and other approaches in these types of devices. As authors reported in [40] they get data communication speed of $120 \mathrm{bps}$ and $72 \mathrm{pJ} / \mathrm{bit}$, meaning that this optical communication is around 500 times less power consuming compared with IEEE 802.15.4 (considering $3.3 \mathrm{~V}$ of voltage supply, $30 \mathrm{~mA}$ of current in radio transmission and $250 \mathrm{kbps}$ of data rate, which is a very big improvement. Moreover, they get $228 \mathrm{pW}$ of standby power which is around 5300 times less than commercial IEEE 802.15.4 chips as CC2530 (with $1.2 \mu \mathrm{W}$ of sleep power at $3 \mathrm{~V}$ ). Therefore, it seems more suitable for very long-term lifetime motes the use of this technology instead of radio links, in terms of power consumption. However, the power of mesh networking is lost and the limitation of the line of sight is imposed. Anyway, the restriction of the power consumption is so strong that, in the case of unattended very 
TABLE II

Iot State of the Art of Very Long-Term Lifetime Extreme Edge Nodes and Their Main Characteristics

\begin{tabular}{|c|c|c|c|c|c|c|c|c|c|c|}
\hline & Power peak & Power standby & Size & Communication & Sensors & Technology & Very long-term lifetime & Application & Core Architecture & Software Support \\
\hline García-Lesta [41] & $600 \mathrm{~mW}$ & $500 \mu \mathrm{W}$ & $\sim \mathrm{cm}^{3}$ & $\begin{array}{c}\text { ZigBee } \\
\text { Xbee Module }\end{array}$ & $\begin{array}{l}\text { Cooper electrodes } \\
\text { capacitive detectors }\end{array}$ & CMOS & $\begin{array}{l}\text { Yes, } \\
\text { solar cells }\end{array}$ & Snail detection & $\begin{array}{c}\text { Arduino FIO } \\
\text { (ATmega328P) }\end{array}$ & Arduino API \\
\hline Lee [40] & $40 \mu \mathrm{W}$ & $11 \mathrm{nW}$ & $1 \mathrm{~mm}^{3}$ & Optical & Temperature, Image & CMOS & $\begin{array}{l}\text { Yes, } \\
\text { solar cells }\end{array}$ & General purpose & $\begin{array}{c}\text { SoC with integrated } \\
\text { Energy and Sensing Manag. }\end{array}$ & $\begin{array}{l}\text { ARM Cortex-M0 } \\
\text { uC libraries }\end{array}$ \\
\hline Quian [43] & $500 \mu \mathrm{W}$ & . & $1 \mu \mathrm{m}^{3}$ & Trigger bit & Infrared & MEMS photoswitch & $\begin{array}{l}\text { Yes, } \\
\text { photoswitches }\end{array}$ & Infrared Digitizer & No processing Core & \\
\hline Rajaram [45] & $2.6 \mathrm{nW}$ & - & $\sim \mathrm{cm}^{2}$ & Trigger bit + Sub-GHzRadio & Infrared & CMOS+photoswitches & $\begin{array}{l}\text { Yes, piezoelectric } \\
\text { generator }\end{array}$ & Infrared Digitizer & CC1310 Wireless MCU & TI SimpleLink IDE \\
\hline Mayer [46] & $55 \mu \mathrm{W}$ & $28 \mu \mathrm{W}$ & $\sim \mathrm{cm}^{2}$ & Trigger bit & $\begin{array}{l}\text { Acoustic MEMS } \\
\text { sensor }\end{array}$ & CMOS & $\begin{array}{l}\text { Yes, microbial } \\
\text { fuel cells }\end{array}$ & $\begin{array}{l}\text { Underwater } \\
\text { sound sensing }\end{array}$ & PIC12LF1552 & Application specific \\
\hline Reger [48] & $5.4 \mathrm{nW}$ & . & $\sim \mathrm{cm}^{2}$ & Trigger bit & Accelerometer & Piezoelectric MEMS+CMOS & Near-zero power & $\begin{array}{l}\text { Acceleration } \\
\text { measurement }\end{array}$ & $\begin{array}{l}\text { No processing Core } \\
\text { (Wake-up element) }\end{array}$ & - \\
\hline Pinrod [49] & $5 \mathrm{nW}$ & . & $322 \mathrm{~mm}^{3}$ & Trigger bit & $\begin{array}{l}\text { Acceleration, magnetic field, } \\
\text { Orientation }\end{array}$ & PZT + NEMS switches & $\begin{array}{l}\text { Yes, piezoelectric } \\
\text { generator }\end{array}$ & Movement sensing & $\begin{array}{l}\text { No processing Core } \\
\text { (Wake-up element) }\end{array}$ & - \\
\hline Zhang [50] & $0.03-0.08 \mathrm{nW}$ & - & $0.017 \mathrm{~mm}^{2}$ & Digital signal: Frequency & Temperature & CMOS & No (no power supply) & $\begin{array}{l}\text { Temperature } \\
\text { sensing }\end{array}$ & Sensing provision & Relied on the Edge \\
\hline Jeong [51] & $12 \mu \mathrm{W}$ & $11 \mathrm{nW}$ & $\sim \mathrm{cm}^{2}$ & Optical & $\begin{array}{c}\text { Acoustic MEMS } \\
\text { sensor }\end{array}$ & CMOS & $\begin{array}{l}\text { No, tens of years } \\
\text { with batteries }\end{array}$ & $\begin{array}{l}\text { Target identification } \\
\text { with sound }\end{array}$ & Dedicated CMOS DSP & Application specific \\
\hline
\end{tabular}

long-term lifetime sensors, the traditional concept of radio communication may be sacrificed.

One step forward in minimizing sensor node architecture is the one presented in [51], where authors present a microsystem for acoustic sensing and object recognition for IoT without wireless communication. The key aspect is that this system is designed for unattended ground sensor nodes. The system is presented as the sensing and processing stage, but no communication hardware is introduced to make this node part of a network. Instead of including communications, authors consider this contribution as a waking-up element for more powerful devices, in case a positive result has been obtained, mainly to deal with false alarms. Waking-up polices are other significant approaches when unattended performance is required. According to [51], this acoustic microsystem consumes $12 \mathrm{~nJ}$ which is enough to work with a $1 \mathrm{~mm}^{2}$ solar panel in indoor light condition (100 lux).

It is clear that radio communications will only be present in the extreme edge when a suitable energy source is available (the mains or an appropriate energy harvester in the order of hundreds of watts) or wake-up policies are implemented.

\section{E. Software Application Development in the Extreme Edge}

In fact, from the point of view of software support upon this extremely constrained sensor layers, the intrinsic nature and requirements of the extreme edge make the development of long-term near-zero-power devices an important challenge that shall be also addressed considering the application context and specific requirements for the target deployment environment. It means that the generalization of functionalities beyond common sensor node development implementation becomes more restricted than in case of the traditional Edge devices.

This is illustrated in Table II, where the comparison of the software support for each type of extreme edge node is also shown, along with the type of core architecture that drives the management of the node (if there is any available). The processing/control elements integrated into most of the verylong-term lifetime devices are very specific to the type of process they are intended for, so similarities along toolkits can be rather encountered from the code-building support tools provided by manufacturers of microcontrollers/DSPs, but not from the Extreme Node itself, as there is not a common well-established high-level programming model for them (unlike software support platforms already available for the Edge, as mentioned before). Thus, application-specific software development is intrinsically performed. Nevertheless, it can be also noticed that some of the solutions can be combined with edge computing strategies right on top of the extreme devices to benefit from these very efficient sensing, triggering and wake-up capabilities.

In particular, the works proposed in [40], [41], [45], and [46] provides trade-off solutions to push a greater subsection of the edge computing and embedded management into the extreme devices, with a winning balance between very long-term lifetime and software application development (considering the potential of creating reduced lightweight libraries for node management in such cases). In the meantime, works as [48]-[50] provide ultra-efficient sensing and triggering capabilities in terms of energy savings for critical deployments where the power supply is a primarily concern. Thus, the potential of these solutions can be indeed exploited by fostering a tied correlation between the distribution of extreme devices with gathering capabilities in the edge (in the form of sensing and triggering actions), so that a complementary energy-efficient computing can be put in place at the bottom of IoT.

\section{F. Actuation in the Extreme Edge}

The extreme edge imposes strong requirements to the devices in terms of power consumption. In this regard, even though actuation is a key issue in the Internet of Things paradigm, in many cases it is restricted to switching on and off other systems with more capabilities in terms of actuation and processing power, or energy budget. In this context, the system is powered off and a trigger event can produce, for example, a digital action when a specific event connected to a physical parameter occurs, as in [49]. Therefore, if actuation is required, special care has to be taken to ensure that the energy budget available is enough to fulfill the requirements of the application in the edge.

\section{G. Influence of Application Requirements Against Lifetime}

As there is an important diversity of possible IoT application scenarios, the associated requirements to meet the specification of the target sensor deployments will ultimately have effects on the lifetime at both node and network levels. Direct and indirect requirements can be highlighted. The former ones are indeed related to the application specifics, such as needed sensing capabilities and number of nodes to be deployed for sensing coverage of the target environment, development and platform costs, the scale of the solution, communication and wireless coverage constraints, mobility, and Quality of Service (QoS) among others; whereas the latter set the intrinsic implications for completion of the requirements, such as the 
type of multi-hop routing strategy (which would have an impact in the long-term network lifetime balance), the application duty cycle, the realization of the power supply and management based on the available sources (in accordance with the scenario constraints), computing and resource usage. They are all going to produce a specific influence on the energy consumption of the sensor nodes and thus on the overall lifetime of the system. As a result, according to the confluence between the application requirements and the resource limitations on the edge and the extreme edge, lifetime maximization techniques can be certainly applied so as to tackle (to a certain extent) the impact of the input design constraints [52].

For instance, Nguyen et al. [53] evaluate the impact of the duty cycle and battery selection on the lifetime, and they verify that the lifetime of a node with $100 \%$ of duty cycle, that is, with no sleep mode activations and radio module always on (with no communication), can go down to less than $80 \mathrm{~h}$ in the extreme study case example with respect to a $25 \%$ of the duty cycle, where almost $200 \mathrm{~h}$ can be achieved (depending on the nominal values of the selected batteries for the experiments). In the meantime, the duty cycle has a relationship with the application requirements. A high degree of mobility among the participant nodes may need increasing the radio activity as the routing strategies would rely on dynamic and reactive mechanisms to find the optimal route to the destination point. It is also interesting to remark from that experimental study that the choice of the type of battery can have an impact of $25 \%$ savings in the lifetime [53], which provides an idea of the importance of the design and implementation stages once the application constraints and requirements are set.

Strategies around opportunistic transmissions, clustering and coverage optimization techniques [52] play a crucial role both to provide an overall balance among the sensor network and to complement the lifetime maximization based on energy awareness designs, including energy harvesting and near-zero power technologies. As shown in Table II, solutions are conceived for accomplishing almost perpetual systems with extremely reduced power consumptions even in active modes, by means of dedicated energy harvesting techniques (and even power management subsystems when possible) to extend the node lifetime depending on the application requirements, as illustrated in the work presented in [40].

\section{REQUIREMENTS FOR REALISTIC IOT DEPLOYMENTS}

From the previous section, a classification for nodes in the edge can be made attending to power consumption. Four types of edge nodes can be distinguished:

1) Edge Nodes: devices that overlap with the limits of the fog. The processing capabilities are in the order of single board computers like Raspberry Pi or BeagleBone Black or even more powerful systems as the NVIDIA Jetson family. The order of magnitude of the power consumption of these nodes is from more than one watt to tens of watts.

2) Motes: Tiny embedded systems with low-end microcontrollers and radio transceivers communication data at hundreds of kbps, as TelosB. These devices consume between hundreds of milliwatts to hundreds of microwatts, depending on the specific hardware architecture

3) Smart Dust type. Integrated devices, usually with solar cells, with sizes in the order of $\mathrm{mm}^{3}$, and with optical communications, more reduced power that radio. These devices consume in the order of microwatts or less.

4) Zero-power sensor nodes: event-driven devices that take energy from the signal to be measured, no requiring in general additional power systems as in the case of the Smart Dust approach. The system is usually consuming near to zero power until the event of interest occurs, waking up the system and supplying the necessary energy to trigger a signal. The consumption of these devices is in the nanowatt domain.

In Figure 3 the different types of edge nodes are plotted considering power consumption and lifetime.

As a result of the information displayed in the previous sections, it is possible to distinguish different domains for the edge devices, from the highest level closer to the fog and the cloud, to the sensor layer close to the signals. Depending on the processing requirements, data will move between these levels accommodating where the processing is taking place.

Attending to the extreme edge, in Table II the very longterm lifetime IoT devices analyzed in the previous section are shown, exhibiting the main characteristics extracted from the research works. A proper combination of harvesting, zero-power sensing, event-driven performance and low power techniques may drive to applications that can last for tens of years, making realistic the realization of the Internet of Things.

The path to realistic IoT edge deployments where autonomy is mandatory is set by the aforementioned approaches. In applications where always-on sensing is needed, and these are a big set in IoT scenarios, zero or near zero-power sensors are one of the keys to allow perpetual or tens of years living systems. Sensors consuming nanowatts with the ability to wake up more powerful devices that are off, will permit the realization of the Internet of Things in the near future.

It is clear that the paradigm shift is taking place in the very bottom of the IoT, to allow the capability of deploying sensor networks that can generate valuable and usable data. This is happening by overcoming what has always been one of the main barriers that made very difficult the explosion of the wireless sensor networks and hence, of the IoT, this is the power consumption and the perpetuity of the devices.

Therefore, the always-on event-driven sensors, that include micro and nano-electromechanical switches instead of electronic circuits, optical communications, wake-up policies with trigger-bit sensors appear as feasible solutions nowadays, and advances in this way are leading the way for the extreme edge of the IoT. It is fair to say that is now more than ever when the IoT together with the wireless sensor networks are in their closest place to be a reality in the people's life.

\section{A. Research Challenges and Open Issues}

An important challenge to be faced with the fast and continuous evolution of IoT is the trade-off analysis between 
very-long-term lifetimes and technology progress, as the extended autonomy of the sensor devices (expected to last for years with the discussed strategies) would certainly cause a confluence of such lifetime with the aging and obsolescence of the deployed pieces, or at least part of the implemented system. This means that, although the sensor node has been conceived to guarantee almost perpetuity, the actual autonomy of the deployment would be truncated by the intrinsic obsolescence problem of the devices. Indeed, the way system design, manufacturing and integration processes are carried out shall also consider how the main target of very-longterm lifetimes will be supported by technologies that take into account longevity-aware strategies. This is not only a concern for the planning and development stages, but for the deployment, commissioning and even the decommissioning process of the overall life cycle of the aimed system.

What strategies and techniques shall be accounted for to provide lifetime vs. longevity-conscious IoT edge and extreme edge deployments? Four main concepts have to be reinforced (at least) during the design, development, implementation, and commissioning of the IoT sensor nodes: Scalability of the solutions, so that the system would evolve and grow depending on the dynamic requirements of the IoT context; reconfigurability/reprogrammability, which allow runtime modifications, updating and replacement of functional blocks of the system; standardization, considering protocols and abstraction layers that provide a well-defined framework for long-term seamless integration of actual and new hardware components; and modularity, which fosters reusability and coexistence of hardware components.

\section{CONCLUSION}

The Internet of Things foreseen at the end of the nineties seems nowadays to breathe stronger due to the advances made in low and zero-power sensing and signal processing. That is the bottom layer of the IoT, the extreme edge, where the things are. Over it, a continuum of different types of devices range from the extreme edge to the fog and the cloud, where data are managed and processed for the good of the humankind.

Researchers seem to have found the right path to very longterm lifetime smart sensors, the key enabling the IoT. It has just started but the first steps in the right direction have been taken. It is now more clear that in the next decade more than 20 billion things deployed will certainly be a reality. That will bring other problems related to privacy or security, and moreover to recycling and trash management of extreme edge devices.

\section{ACKNOWLEDGMENT}

The authors and specifically Jorge Portilla would like to thank the "José Castillejo" program for junior postdoc research stays, funded by the Ministry of Education, Culture and Sports of the Government of Spain, grant number CAS17/00259, stay during which this paper has been written.

\section{REFERENCES}

[1] K. Ashton, (2009). That 'Internet of Things' Thing-2009-06-22-Page 1RFID Journal. [Online]. Available: http://www.rfidjournal.com/articles/ view4986
[2] M. Weiser, "The computer for the 21st century," SIGMOBILE Mobile Comput. Commun. Rev., vol. 3, no. 3, pp. 3-11, Jul. 1999.

[3] (2016). IoT: Number of Connected Devices Worldwide 2012-2025 Statista. [Online]. Available: https://www.statista.com/statistics/471264/ iot-number-of-connected-devices-worldwide

[4] Internet of Things Forecast-Ericsson. Accessed: Jul. 5, 2018. [Online]. Available: https://www.ericsson.com/en/mobility-report/internet-ofthings-forecast

[5] Gartner Connected 'Things'. [Online]. Available: https://www.gartner. com/newsroom/id/3598917

[6] Market Pulse Report, Internet of Things (IoT), GrowthEnabler, Bengaluru, India, 2017.

[7] D. Bilgeri, V. Brandt, M. Lang, J. Tesch, and M. Weinberger, "The IoT business model builder," Bosch Softw. Innov. GmbH, White Paper, 2015.

[8] M. Chiang and T. Zhang, "Fog and IoT: An overview of research opportunities," IEEE Internet Things J., vol. 3, no. 6, pp. 854-864, Dec. 2016.

[9] P. Mell and T. Grance, "The NIST definition of cloud computing," Nat. Inst. Standards Technol., Gaithersburg, MD, USA, Tech. Rep., 2011.

[10] W. Shi, J. Cao, Q. Zhang, Y. Li, and L. Xu, "Edge computing: Vision and challenges," IEEE Internet Things J., vol. 3, no. 5, pp. 637-646, Oct. 2016.

[11] F. Bonomi, R. Milito, J. Zhu, and S. Addepalli, "Fog computing and its role in the Internet of Things," in Proc. 1st Ed. MCC Workshop Mobile Cloud Comput. New York, NY, USA, 2012, pp. 13-16.

[12] M. Iorga, L. Feldman, R. Barton, M. J. Martin, N. S. Goren, and C. Mahmoudi, "Fog computing conceptual model," Nat. Inst. Standards Technol., Gaithersburg, MD, USA, Tech. Rep., Mar. 2018.

[13] G. Premsankar, M. Di Francesco, and T. Taleb, "Edge computing for the Internet of Things: A case study," IEEE Internet Things J., vol. 5, no. 2, pp. 1275-1284, Apr. 2018.

[14] H. El-Sayed et al., "Edge of things: The big picture on the integration of edge, IoT and the cloud in a distributed computing environment," IEEE Access, vol. 6, pp. 1706-1717, 2017.

[15] J. S. Preden, K. Tammemäe, A. Jantsch, M. Leier, A. Riid, and E. Calis, "The benefits of self-awareness and attention in fog and mist computing," Computer, vol. 48, no. 7, pp. 37-45, Jul. 2015.

[16] T. Karnik et al., "A cm-scale self-powered intelligent and secure IoT edge mote featuring an ultra-low-power SoC in $14 \mathrm{~nm}$ tri-gate CMOS," in IEEE Int. Solid-State Circuits Conf. (ISSCC) Dig. Tech. Papers, Feb. 2018, pp. 46-48.

[17] X. Sun and N. Ansari, "EdgeIoT: Mobile edge computing for the Internet of Things," IEEE Commun. Mag., vol. 54, no. 12, pp. 22-29, Dec. 2016.

[18] Y. Jararweh, A. Doulat, O. AlQudah, E. Ahmed, M. Al-Ayyoub, and E. Benkhelifa, "The future of mobile cloud computing: Integrating cloudlets and mobile edge computing," in Proc. 23rd Int. Conf. Telecommun. (ICT), May 2016, pp. 1-5.

[19] Z. Xiao, H. B. Lim, and L. Ponnambalam, "Participatory sensing for smart cities: A case study on transport trip quality measurement," IEEE Trans. Ind. Informat., vol. 13, no. 2, pp. 759-770, Apr. 2017.

[20] F. Javed, M. K. Afzal, M. Sharif, and B.-S. Kim, "Internet of Things (IoT) operating systems support, networking technologies, applications, and challenges: A comparative review," IEEE Commun. Surveys Tuts. vol. 20, no. 3, pp. 2062-2100, 3rd Quart., 2018.

[21] J. Zornoza, G. Mujica, J. Portilla, and T. Riesgo, "Merging smart wearable devices and wireless mesh networks for collaborative sensing," in Proc. 32nd Conf. Design Circuits Integr. Syst. (DCIS), Nov. 2017, pp. $1-6$.

[22] J. Gascon-Samson, M. Rafiuzzaman, and K. Pattabiraman, "ThingsJS: Towards a flexible and self-adaptable middleware for dynamic and heterogeneous IoT environments," in Proc. 4th Workshop Middleware Appl. Internet Things, New York, NY, USA, 2017, pp. 11-16.

[23] K. Hong, D. Lillethun, U. Ramachandran, B. Ottenwälder, and B. Koldehofe, "Mobile fog: A programming model for large-scale applications on the Internet of Things," in Proc. 2nd ACM SIGCOMM Workshop Mobile Cloud Comput., New York, NY, USA, 2013, pp. 15-20.

[24] H. Omar, "Intelligent traffic information system based on integration of Internet of Things and agent technology," Int. J. Adv. Comput. Sci. Appl., vol. 6, Feb. 2015, pp. 37-43.

[25] C. Savaglio, G. Fortino, and M. Zhou, "Towards interoperable, cognitive and autonomic IoT systems: An agent-based approach," in Proc. IEEE 3rd World Forum Internet Things (WF-IoT), Dec. 2016, pp. 58-63.

[26] C. Pallasch et al., "Edge powered industrial control: Concept for combining cloud and automation technologies," in Proc. IEEE Int. Conf. Edge Comput. (EDGE), Jul. 2018, pp. 130-134. 
[27] N.-N. Dao, Y. Lee, S. Cho, E. Kim, K.-S. Chung, and C. Keum, "Multitier multi-access edge computing: The role for the fourth industrial revolution," in Proc. Int. Conf. Inf. Commun. Technol. Converg. (ICTC), Oct. 2017, pp. 1280-1282.

[28] K. J. Singh and D. S. Kapoor, "Create your own Internet of Things: A survey of IoT platforms," IEEE Consum. Electron. Mag., vol. 6, no. 2, pp. 57-68, Apr. 2017.

[29] J. Polastre, R. Szewczyk, and D. Culler, "Telos: Enabling ultra-low power wireless research," in Proc. 4th Int. Symp. Inf. Process. Sensor Netw. (IPSN), Apr. 2005, pp. 364-369.

[30] J. Hill, R. Szewczyk, A. Woo, S. Hollar, D. Culler, and K. Pister, "System architecture directions for networked sensors," ACM SIGOPS Operating Syst. Rev., vol. 35, no. 5, pp. 93-104, Nov. 2000.

[31] A. Dunkels, B. Gronvall, and T. Voigt, "Contiki-A lightweight and flexible operating system for tiny networked sensors," in Proc. 29th Annu. IEEE Int. Conf. Local Comput. Netw., Nov. 2004, pp. 455-462.

[32] A. Prayati, C. Antonopoulos, T. Stoyanova, C. Koulamas, and G. Papadopoulos, "A modeling approach on the TelosB WSN platform power consumption," J. Syst. Softw., vol. 83, no. 8, pp. 1355-1363, Aug. 2010.

[33] A. E. Xhafa, B. Campbell, and S. Hosur, "Towards a perpetual wireless sensor node," in Proc. IEEE SENSORS, Nov. 2013, pp. 1-4.

[34] J. Portilla, A. de Castro, E. de la Torre, and T. Riesgo, "A modular architecture for nodes in wireless sensor networks," J. Universal Comput. Sci., vol. 12, no. 3, pp. 328-339, 2006.

[35] C. Pham, "Communication performances of IEEE 802.15.4 wireless sensor motes for data-intensive applications: A comparison of WaspMote, Arduino MEGA, TelosB, MicaZ and iMote2 for image surveillance," J. Netw. Comput. Appl., vol. 46, pp. 48-59, Nov. 2014.

[36] J. M. Kahn, R. H. Katz, and K. S. J. Pister, "Next century challenges: Mobile networking for "smart dust'," in Proc. 5th Annu. ACM/IEEE Int. Conf. Mobile Comput. Netw. (MobiCom), New York, NY, USA, 1999, pp. 271-278.

[37] H. Hejazi, H. Rajab, T. Cinkler, and L. Lengyel, "Survey of platforms for massive IoT," in Proc. IEEE Int. Conf. Future IoT Technol. (Future IoT), Jan. 2018, pp. 1-8.

[38] S. Basu, L. Duch, M. Peón-Quirós, D. Atienza, G. Ansaloni, and L. Pozzi, "Heterogeneous and inexact: Maximizing power efficiency of edge computing sensors for health monitoring applications," in Proc. IEEE Int. Symp. Circuits Syst. (ISCAS), May 2018, pp. 1-5.

[39] N. S. Alhassoun, "Energy optimization for perpetual IoT networks of heterogeneous sensors," in Proc. 8th Int. Green Sustain. Comput. Conf. (IGSC), Oct. 2017, pp. 1-3.

[40] Y. Lee et al., "A modular $1 \mathrm{~mm}^{3}$ die-stacked sensing platform with low power $\mathrm{I}^{2} \mathrm{C}$ inter-die communication and multi-modal energy harvesting," IEEE J. Solid-State Circuits, vol. 48, no. 1, pp. 229-243, Jan. 2013.

[41] D. García-Lesta, D. Cabello, E. Ferro, P. López, and V. M. Brea, "Wireless sensor network with perpetual motes for terrestrial snail activity monitoring," IEEE Sensors J., vol. 17, no. 15, pp. 5008-5015, Aug. 2017.

[42] P. Harpe, H. Gao, R. van Dommele, E. Cantatore, and A. H. M. van Roermund, "A $0.20 \mathrm{~mm}^{2} 3 \mathrm{nW}$ signal acquisition ic for miniature sensor nodes in $65 \mathrm{~nm}$ CMOS," IEEE J. Solid-State Circuits, vol. 51, no. 1, pp. 240-248, Jan. 2016.

[43] Z. Qian, S. Kang, V. Rajaram, C. Cassella, N. E. McGruer, and M. Rinaldi, "Zero-power infrared digitizers based on plasmonically enhanced micromechanical photoswitches," Nature Nanotechnol., vol. 12, pp. 969-973, Sep. 2017.

[44] U. Zaghloul and G. Piazza, "Sub-1-volt piezoelectric nanoelectromechanical relays with millivolt switching capability," IEEE Electron Device Lett., vol. 35, no. 6, pp. 669-671, Jun. 2014.

[45] V. Rajaram, Z. Qian, S. Kang, N. E. McGruer, and M. Rinaldi, "MEMS-based near-zero power infrared wireless sensor node," in Proc. IEEE Micro Electro Mech. Syst. (MEMS), Jan. 2018, pp. 17-20.

[46] P. Mayer, M. Magno, and L. Benini, "Combining microbial fuel cell and ultra-low power event-driven audio detector for zero-power sensing in underwater monitoring," in Proc. IEEE Sensors Appl. Symp. (SAS), Mar. 2018, pp. 1-6.

[47] R. H. Olsson, R. B. Bogoslovov, and C. Gordon, "Event driven persistent sensing: Overcoming the energy and lifetime limitations in unattended wireless sensors," in Proc. IEEE SENSORS, Oct./Nov. 2016, pp. 1-3.

[48] R. W. Reger et al., "Near-zero power accelerometer wakeup system," in Proc. IEEE SENSORS, Oct./Nov. 2017, pp. 1-3.

[49] V. Pinrod et al., "Zero-power sensors with near-zero-power wakeup switches for reliable sensor platforms," in Proc. IEEE 30th Int. Conf. Micro Electro Mech. Syst. (MEMS), Jan. 2017, pp. 1236-1239.
[50] R. Zhang, S. Fan, and L. Geng, "A near-zero-power temperature sensor with $\pm 0.24{ }^{\circ} \mathrm{C}$ inaccuracy using only standard CMOS transistors for IoT applications," in Proc. IEEE Int. Symp. Circuits Syst. (ISCAS), May 2018, pp. 1-4.

[51] S. Jeong et al., "Always-on 12-nW acoustic sensing and object recognition microsystem for unattended ground sensor nodes," IEEE J. SolidState Circuits, vol. 53, no. 1, pp. 261-274, Jan. 2018.

[52] H. Yetgin, K. T. K. Cheung, M. El-Hajjar, and L. Hanzo, "Networklifetime maximization of wireless sensor networks," IEEE Access, vol. 3, pp. 2191-2226, 2015

[53] H. A. Nguyen, A. Förster, D. Puccinelli, and S. Giordano, "Sensor node lifetime: An experimental study," in Proc. IEEE Int. Conf. Pervasive Comput. Commun. Workshops (PERCOM Workshops), Mar. 2011, pp. 202-207.

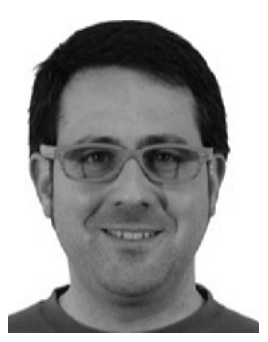

Jorge Portilla (M'09-SM'18) received the M.Sc. degree in physics from the Universidad Complutense de Madrid, Madrid, Spain, in 2003, and the Ph.D. degree in electronic engineering from the Universidad Politécnica de Madrid (UPM), Madrid, Spain, in 2010.

He was a Visiting Researcher with the Industrial Technology Research Institute, Hsinchu, Taiwan, in 2008, and also with the National Taipei University of Technology (Taipei Tech), Taipei, Taiwan, in 2018, working on wireless sensor networks hardware platforms and network clustering techniques. He is currently an Assistant Professor Tenured with Universidad Politécnica de Madrid. He carries out his research activity within the Centro de Electrónica Industrial, belonging to the UPM. His research interests are focused on wireless sensor networks, Internet of Things, digital embedded systems, and reconfigurable FPGA-based embedded systems. He has participated in more than 30 funded research projects, including European Union FP7 and H2020 projects, and Spain Government funded projects, as well as private industry funded projects, mainly related to wireless sensor networks and Internet of Things. He has numerous publications in prestigious international conferences as well as in journals with impact factor.

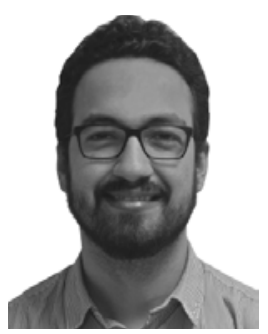

Gabriel Mujica (M'18) received the Ph.D. degree in industrial electronics engineering from the Center of Industrial Electronics, Universidad Politécnica de Madrid. He is a Post-Doctoral Research Member with the Center of Industrial Electronics, Universidad Politécnica de Madrid, where he is mainly involved in the area of networked embedded systems and wireless sensor networks (WSN).

$\mathrm{He}$ has participated in different national and European research projects (including Horizon 2020 projects), related to the development and optimization of WSN, as well as the integration of heterogeneous hardware, software, and communication technologies for wireless distributed systems, with a particular focus on the performance evaluation of sensor platforms under real deployment and commissioning. In this way, he has authored several contributions in high-impact conferences and journals. He has collaborated in the organization of research tutorials and seminars, and as a reviewer for several international conferences and journals (IEEE and Springer). Moreover, his visiting research stay at Trinity College Dublin strengthened the vision and applicability of IoT technologies for smart and sustainable cities, leveraging collaborations in the area of distributed systems within such contexts. Currently, his main research interests are related to multi-hop distributed networks, hardware-software co-design and protocols for embedded systems in smart urban application scenarios. 


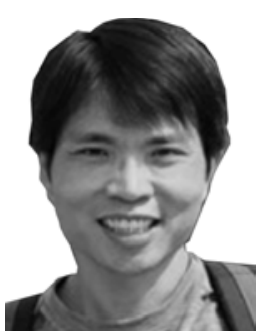

Jin-Shyan Lee (M'10-SM'11) received the B.S. degree in mechanical engineering from the National Taiwan University of Science and Technology, Taipei, Taiwan, in 1997, and the M.S. and Ph.D. degrees in electrical and control engineering from National Chiao Tung University, Hsinchu, Taiwan, in 1999 and 2004, respectively.

From 2005 to 2009, he was a Researcher with the Information and Communications Research Laboratory, Industrial Technology Research Institute, Zhudong, Taiwan. He then joined the Department of Electrical Engineering, National Taipei University of Technology, Taipei, where he is currently an Associate Professor. From 2003 to 2004 and from 2015 to 2016, he was a Visiting Scholar with the Department of Electrical and Computer Engineering, New Jersey Institute of Technology, Newark, NJ, USA. His current research interests include wireless sensor networks, Petri nets, intelligent systems, and automatic control. He is a member of the Technical Committee on Environmental Sensing, Networking and DecisionMaking and the Technical Committee on Discrete Event Systems of the IEEE Systems, Man, and Cybernetics Society. Dr. Lee was a recipient of the 2015 Outstanding Youth Award from the Taiwan Association of Systems Science and Engineering, the 2010 Early Career Award from the IEEE Industrial Electronics Society, the 2008 Youth Automatic Control Engineering Award from the Chinese Automatic Control Society, and the 2004 International Scholarship from the Society of Instrument and Control Engineers. He has been the Track Chair of the IEEE International Conference on Industrial Electronics and Applications since 2010.

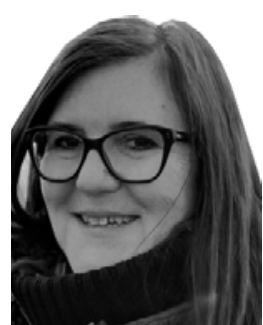

Teresa Riesgo (M'96) received the M.Sc. and Ph.D. degrees in electrical engineering from the Universidad Politécnica de Madrid (UPM), Madrid, Spain, in 1989 and 1996, respectively.

Since 2003, she has been a Full Professor of Electronics at UPM. Her research interests are focused on embedded system design, wireless sensor networks, Internet of Things, configurable systems, and power estimation in digital systems. She has participated and acted as the main researcher in more than 60 national and European research, development, and innovation projects, almost always in collaboration with the industry As a result of her research activity, she has published more than 200 scientific documents in journals and international congress proceedings. She has been the Director of the Center for Industrial Electronics, the Deputy Director of Research and International Relations at the ETSII-UPM, and, more recently, the Director of the Department of Automation, Electrical and Electronic Engineering and Industrial Computing until 2018. Since 2003, she has been the Representative of the UPM in the TIME Network (Top Industrial Managers in Europe) and a member of its management committee. She participates regularly in evaluation panels of national projects and EU framework programs. In 2018, she was appointed as the General Director of Research, Development and Innovation, a position under the Ministry of Science, Innovation and Universities of the Government of Spain. 